Article

\title{
Experimental Assessment of a Centralised Controller for High-RES Active Distribution Networks
}

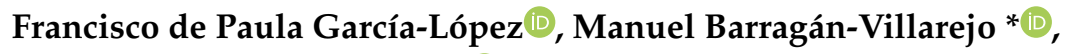 \\ Alejandro Marano-Marcolini $\mathbb{D}^{\mathbb{D}}$, José María Maza-Ortega and José Luis Martínez-Ramos \\ Department of Electrical Engineering, Universidad de Sevilla, Camino de los Descubrimientos s/n, \\ 41092 Seville, Spain; fdpgarcia@us.es (F.d.P.G.-L.); alejandromm@us.es (A.M.-M.); jmmaza@us.es (J.M.M.-O.); \\ camel@us.es (J.L.M.-R.) \\ * Correspondence: manuelbarragan@us.es; Tel.: +34-954-481-281
}

Received: 31 October 2018; Accepted: 28 November 2018; Published: 1 December 2018

check for updates

\begin{abstract}
This paper assesses the behaviour of active distribution networks with high penetration of renewable energy sources when the control is performed in a centralised manner. The control assets are the on-load tap changers of transformers at the primary substation, the reactive power injections of the renewable energy sources, and the active and reactive power exchanged between adjacent feeders when they are interconnected through a DC link. A scaled-down distribution network is used as the testbed to emulate the behaviour of an active distribution system with massive penetration of renewable energy resources. The laboratory testbed involves hardware devices, real-time control, and communication infrastructure. Several key performance indices are adopted to assess the effects of the different control actions on the system's operation. The experimental results demonstrate that the combination of control actions enables the optimal integration of a massive penetration of renewable energy.
\end{abstract}

Keywords: active distribution network; laboratory testbed; renewable energy sources; DC link; centralised control

\section{Introduction}

Massive penetration of renewable energy sources (RES) is unstoppable nowadays because of the need to reduce the dependency of fossil fuels. This new technology of generation assets is being deployed in small units within medium voltage (MV) and low voltage (LV) distribution systems, the so-called distributed generation, in contrast to the conventional connections of large-scale power plants to high voltage (HV) systems. The drivers behind this change in the generation paradigm are threefold: technical because of the maturity of the technology [1], economical due to the related cost reduction [2], and social because of the citizen involvement in decarbonising the electrical consumption [3].

The traditional operation of radial distribution systems cannot be maintained in cases where there is very high RES penetration, because the design of these systems has been done to cope with power flows from primary and secondary substations to the final users [4]. The problems that RES may create have been profusely described in specialised literature [5], for example, higher simultaneity coefficients, reverse power flows, out of control nodal voltages, power quality deterioration, increase of short-circuit power, etc. These technical problems can be released using conventional network reinforcement strategies ranging from increasing the cross-section of existing lines to installing new lines and/or power transformers. However, it has to be questioned as to whether this is the best solution considering the increases in cost and connection time [6] as well as the spare capacity of the 
new assets over a large number of hours per year [7]. Therefore, new alternatives must be explored to overcome the shortcomings related to this Fit \& Forget approach.

Several active network operation approaches have been proposed recently. In general, these can be classified according to the following characteristics: the control assets used to optimise the network operation, the applied control algorithms, and the testing procedure used to validate their performance.

Control assets: Regarding the first issue, HV/MV transformers equipped with on-load tap changers (OLTCs) and step voltage regulators were proposed in [8]. In addition, RES may also contribute to voltage regulation and congestion management by resorting to curtailment [9] or even by using adequate reactive power injections [10-12]. Most of the major RES inverter manufacturers include the possibility of controlling the reactive power in order to fulfil the grid codes imposed by the system operators (either distribution system operators (DSOs) or transmission system operators (TSOs)). These grid codes are becoming more and more restrictive and they include minimal technical requirements for RES connections to occur [13,14], including, among others, voltage regulation issues by means of reactive power injection.It is important to mention that most of the active operation approaches consider several control assets that are managed in a coordinated manner, for example, HV/MV, OLTC, and RES [15-17]; HV/MV, OLTC, and energy storage systems [18,19]; and HV/MV, OLTC, RES reactive power injection, and direct current (DC) links [20].

Control methodology: The active management solutions can be broadly classified into centralised, distributed, and local methodologies. The centralised approaches rely on a control centre in charge of computing the optimal setpoints for all the control assets that considers the available network measurements [11]. The main drawback of this approach is the need for an extensive communication system. Therefore, this solution is suitable for MV distribution systems because of two reasons. On the one hand, the cost of the communication infrastructure is marginal with respect to the cost of the large RES units (in the range of several MVA). On the other hand, nowadays, utilities are equipped with centralised Advanced Demand Management Systems (ADMS) which incorporate monitoring and automation functionalities. However, it should be considered that a failure of part of the communication infrastructure may deteriorate the performance of the controller. For this reason, advanced control strategies providing enhanced system resilience can be found in the specialised literature [21]. Local approaches are just the opposite because the actions taken by the control assets are calculated based on local measurements [10,12,16,22], and therefore, they are suitable for LV distribution systems. Distributed methods can be considered a compromise between the previous alternatives as they have several advantages related to robustness and scalability [23,24].

Testing methodology: The methodologies are usually validated by applying steady-state simulations that consider the daily load and generation profiles. However, other proposals use real-time digital simulators [23] and power hardware-in-the-loop platforms [19].

The results obtained by some of the previous control approaches can be summarised as follows. In [17], a 32\% reduction of power losses was reached by using an adequate RES reactive power injection. After including the OLTC as an additional control asset, [16] reported an extra 7\% reduction in total daily energy losses. Finally, a similar approach that considered the actual capability curves of the RES units achieved a $14 \%$ reduction in power losses [12].

This paper tests the use of centralised control of active assets to manage MV distribution networks with a massive RES penetration. An Optimal Power Flow (OPF) is used in the centralised control to compute the optimal setpoints for three kinds of control assets: (1) HV/MV-transformer OLTCs, (2) RES reactive power injections, and (3) active and reactive power through DC link meshing radial feeders. A high-RES but realistic load/generation scenario is analysed that considers some test cases involving different sets of control assets with the aim of evaluating their performance. These test cases are implemented in a laboratory scaled-down active distribution network including hardware devices, controllers, communication infrastructure, and a real-time monitoring system, as presented in [25]. This testbed can be used to evaluate practical implementation issues of any centralised control algorithm related to the applied control strategy, the required data field, the communication 
systems, etc., as a step prior to field deployment. Therefore, the main contribution of this paper is the experimental validation of the centralised controller proposed in [20] within an updated version of the testbed described in [25] in which an OLTC transformer, a DC link, and a new control scheme and communication system are incorporated.

The paper is organised as follows. In Section 2, a description of the centralised control to manage high-RES active distribution networks is presented. In Section 3, the benchmark distribution network is described in detail, including its main components and how they are represented in the laboratory scaled-down testing platform. Section 4 depicts and analyses the system's performance in different test cases, comparing them in a quantitative manner by means of key performance indices (KPIs). Finally, Section 5 closes with the main conclusions.

\section{Proposed Centralised Control}

Smart grids are characterized by extensive measurement, automation, and communication infrastructures which allows a safe and optimized network operation that takes advantage of centralised ADMSs. The main role of any ADMS in this environment is to concentrate the field data to extract the required information about the network status and, in cases where control assets are in operation, compute and send the required control actions to optimize the network operation according to a given criterion.

Figure 1 depicts this centralised control approach. First, the smart meters are in charge of measuring the load demanded by industrial $\left(P_{i l}\right.$ and $\left.Q_{i l}\right)$ and residential $\left(P_{h l}\right.$ and $\left.Q_{h l}\right)$ clients. In addition, the RES active power injections, such as the wind turbine (WT) and photovoltaic (PV) plants, $P_{w t}$ and $P_{p v}$, respectively, are measured.

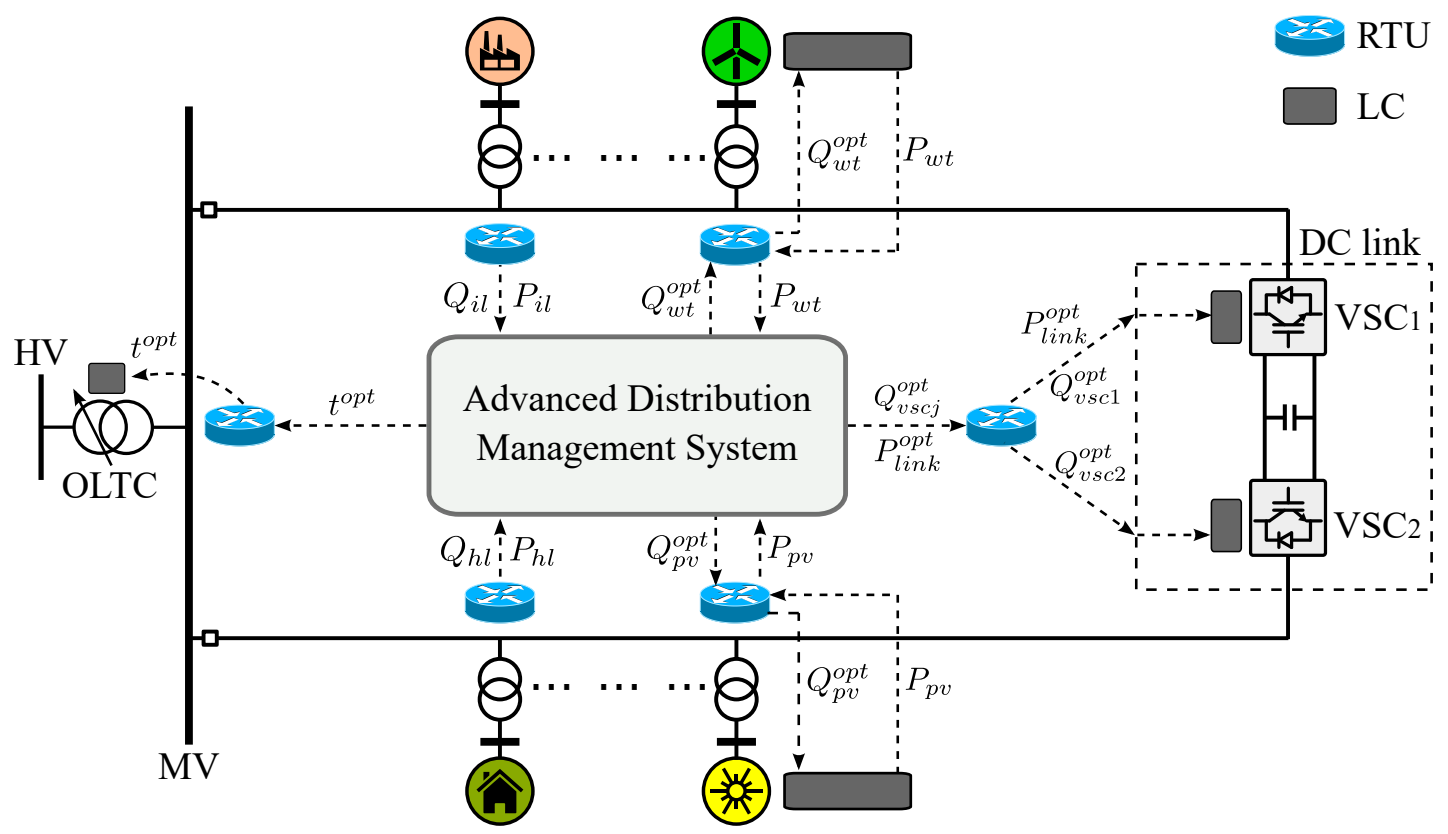

Figure 1. Architecture of the centralised control of an active distribution system.

All field data are sent to the ADMS by means of Remote Terminal Units (RTUs) at regular time intervals (typically 5 to $15 \mathrm{~min}$ ). Considering all this information, it is possible to compute setpoints for the installed control assets using an OPF to optimize any technical or economic objective. This paper considers the following control assets:

- $\quad$ RES, which can regulate the reactive power injections $Q_{w t}^{o p t}$ and $Q_{p v}^{o p t}$.

- Transformer OLTCs, which can adjust the tap position $t^{\text {opt }}$.

- A DC link, which is composed of two Voltage Source Converters (VSCs) in a back-to-back topology connecting two radial feeders. This device can regulate the active power flow between the feeders, 
$P_{\text {link }}^{o p t}$, and two independent reactive power injections, $Q_{v s c j}^{o p t}$. It is important to point out that the DC link is an interesting control asset with proven capability to reduce the network active power losses, maximize the penetration of RES, improve the network voltage profiles, and avoid branch saturations $[20,26]$.

On the other hand, the selected OPF objective is to minimize the active power losses of the system to take advantage of the already available control assets to optimise the operation of the distribution grid, which leads to the following formulation:

$$
\min _{\mathbf{x}} P_{\text {loss }}(\mathbf{x}, \mathbf{y})
$$

where $\mathbf{x}$ is the set of control variables $\left(P_{l i n k}^{o p t}, Q_{v s c j^{\prime}}^{o p t} Q_{w t, p v}^{o p t}, t^{o p t}\right)$ and $\mathbf{y}$ is the set of load and generation power injections for a given time interval $\left(P_{i l}, Q_{i l}, P_{h l}, Q_{h l}, P_{w t}, P_{p v}\right)$.

The optimization problem is completed by including the relevant constraints. First, the network operational limits have to be considered. The voltages and currents of the sets of buses, $\mathcal{N}$, and branches, $\mathcal{B}$, have to be within the regulatory boundaries, $\left[V_{i}^{\min }, V_{i}^{\max }\right]$, and below the cable ampacities, $I_{b}^{\max }$, respectively, as stated in (2) and (3):

$$
\begin{aligned}
V_{i}^{\text {min }} & \leq V_{i} \leq V_{i}^{\text {max }} \quad \forall i \in \mathcal{N}, \\
0 & \leq I_{b} \leq I_{b}^{\text {max }} \quad \forall b \in \mathcal{B} .
\end{aligned}
$$

Second, the OLTC tap has to be within the limits and the apparent power levels of the RES and DC-link VSCs have to be below their rated capability according to (4)-(6):

$$
\begin{gathered}
t^{\text {min }} \leq t^{o p t} \leq t^{\text {max }}, \\
S_{p v, w t} \leq S_{p v, w w}^{r a t}, \\
S_{D C l i n k} \leq S_{D C \text { clink }}^{r a t} .
\end{gathered}
$$

Finally, other constraints which are included in the OPF are the active and reactive bus power balances and the power constraints that model the DC link behaviour, which can be found in [26].

\section{Laboratory Testing Platform}

The objective of building the laboratory testing platform was to faithfully represent the real behaviour of an active distribution system including all of its components to asses the performance of the centralised control strategy outlined in Section 2. In this way, the testing platform was built based on the MV benchmark distribution network proposed by the International Council on Large Electric Systems (CIGRE in french) Task Force C06.04.02 devoted to study the RES integration in MV networks [27]. The main reasons that motivated the selection of this system are detailed below:

- First, this network is based on an actual MV German distribution system, fulfilling the proposed objective of the laboratory testing platform described above.

- Second, an important RES penetration is integrated into the network.

- Third, all the network data, including topology, parameters of lines and cables, loads, RES, and their corresponding daily load/generation curves are available and are well documented.

- Fourth, the benchmark network includes a DC link, a key component of the future active distribution system with high RES penetration.

The next subsections present the MV benchmark distribution system and its scaled-down version built in the laboratory for testing purposes, including the implemented control scheme and the communication infrastructure designed to operate the system as a flexible platform to evaluate the benefits of active distribution networks. 


\subsection{Benchmark Distribution Network}

A one-line diagram of the benchmark distribution system is shown in Figure 2 which is composed of two radial subsystems departing from a primary substation where a 40 MVA $110 / 20 \mathrm{kV}$ transformer equipped with an OLTC is installed. The total network comprises 14 buses grouped in two radial feeders: 11 buses for subsystem 1 and 3 buses for subsystem 2 . The total line length of subsystem 1 is about $15 \mathrm{~km}$, while subsystem 2 is just $8 \mathrm{~km}$. In addition, different types of load, involving industrial and domestic customers as well as a large amount of RES, are connected into the different buses. Although [27] considered different types of RES, this work exclusively included PV and WT plants because its current maturity foresees that they will be massive deployed in upcoming years. In addition, the benchmark network includes a DC link to connect both radial subsystems between nodes N8 and N14.

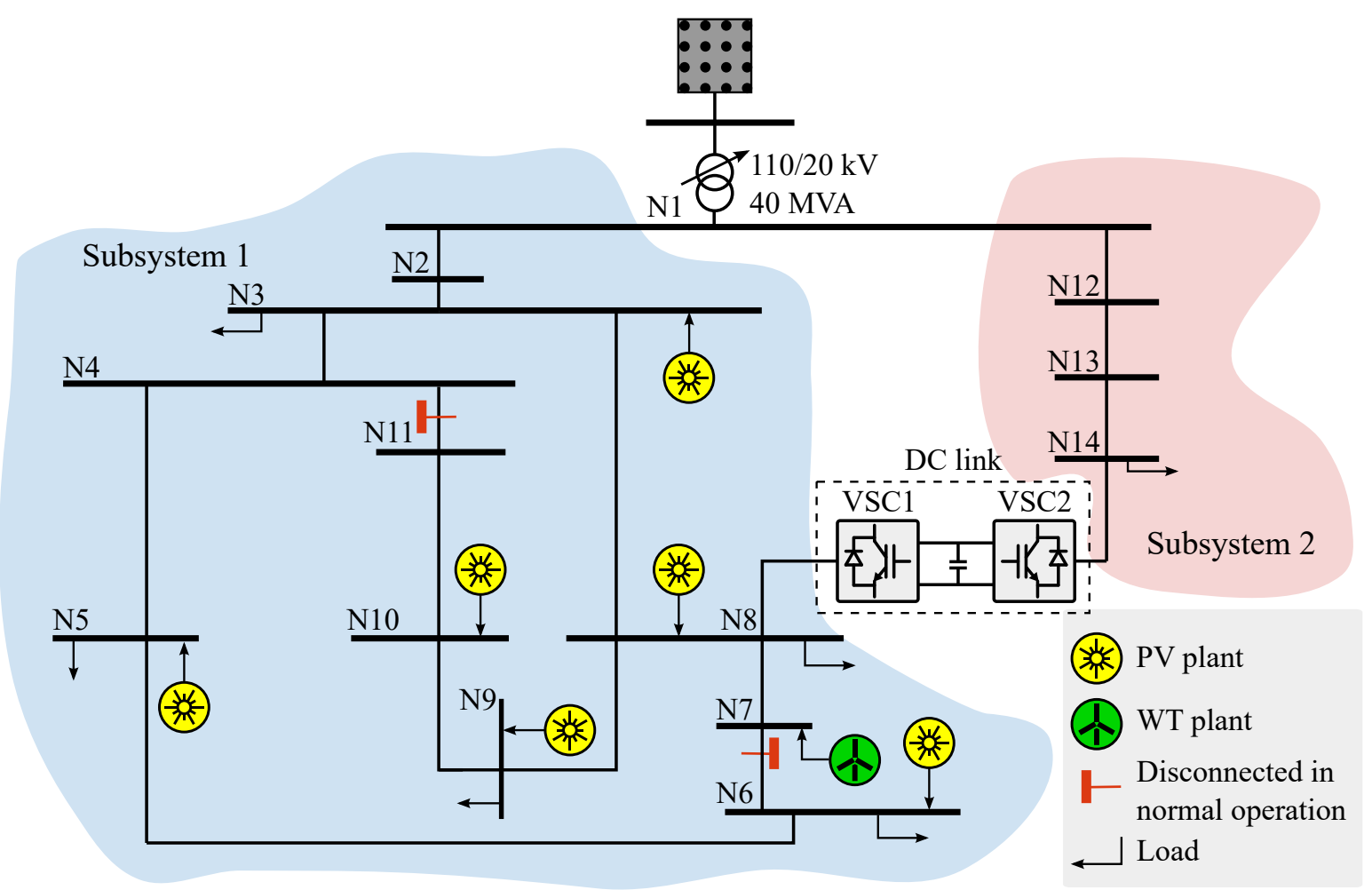

Figure 2. The medium voltage (MV) benchmark distribution network proposed by the CIGRE Task Force C06.04.02.

The 24-h profiles of the total loads and RES of subsystems 1 and 2 are depicted in Figure 3. It is interesting to point out that subsystem 1 was more loaded than subsystem 2. Moreover, most RES were located within subsystem 1 which partially compensate for its higher load with this local generation. It is also worth noting that, in order to analyse a case with a massive RES penetration, the generation was multiplied by 4 and 400 in the case of the WT and PV plants, respectively, with respect to the scenario described in [27]. In this way, the peak generation of the RES units and the peak demands of the loads during the day were established at $0.446 \mathrm{pu}$ and $0.381 \mathrm{pu}$, respectively (the base power of the MV system was $100 \mathrm{MVA}$ ). The ratio between the peak generation and the peak demand was equal to 1.1724 - a scenario of high-RES penetration. 

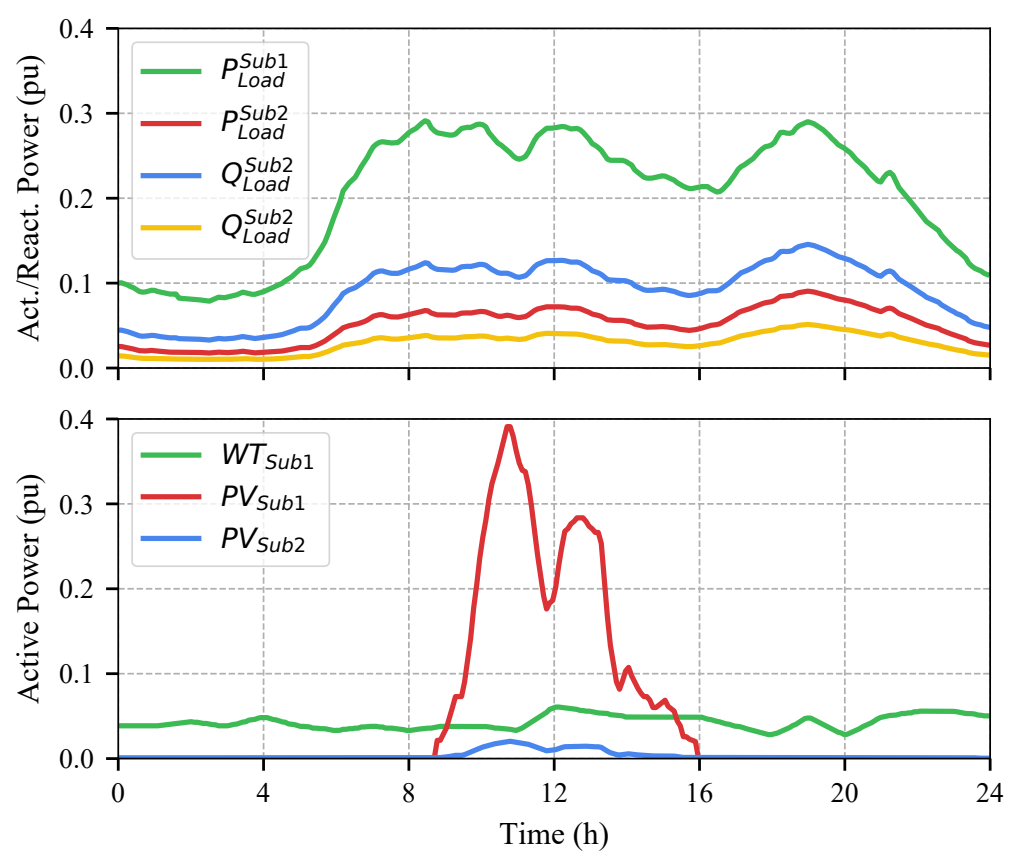

Figure 3. Top: Daily profile of the total loads in subsystems 1 and 2; Bottom: Daily profile of the total WT and PV generation in subsystems 1 and 2.

\subsection{Laboratory Scaled-Down Distribution Network}

This subsection provides a brief outline of the components and functionalities of the scaled-down testbed used to validate the benefits of the centralised controller. Basically, this hardware test rig, depicted in Figure 4, is a three-phase scaled-down $400 \mathrm{~V}$ (base/rated voltage) and $100 \mathrm{kVA}$ (base/rated power) representation of the MV benchmark network described in Section 3.1 which is composed of the following components:

- Distribution network branches: The electrical lines of both scaled-down subsystems are represented by a lumped parameter model comprising the series resistor and reactor. The per unit values of these impedances are identical to those of the actual MV system. Therefore, the original line $\mathrm{R} / \mathrm{X}$ ratios and equivalent lengths are maintained, leading to similar per unit voltage drops and power losses. Table 1 collects the exact values of the resistors and reactors used in the scaled-down network.

- Omnimode Load Emulators (OLEs): These are the building blocks that are responsible for representing any load, generator, or a combination of the two connected to any network node. Basically, each OLE is a VSC with a local controller (LC) whose AC and DC sides are connected to a scaled-down network node and a common DC bus, respectively, as shown in Figure 4. The VSC is a three-phase, three-wire, two-level insulated gate bipolar transistor (IGBT) VSC, rated at $400 \mathrm{~V}$, $20 \mathrm{kVA}$ with a switching frequency of $10 \mathrm{kHz}$. LCL coupling filters are used to connect the AC-side of the VSC to the scaled-down network. The inductors and the capacitor have the following ratings: $\mathrm{L} 1=\mathrm{L} 2=2.5 \mathrm{mH}$ and $\mathrm{C}=1 \mu \mathrm{F}$. Note that all of the OLEs share a common DC bus which is regulated by an extra balancing VSC rated to $100 \mathrm{kVA}$. This is directly connected to the LV laboratory network by its AC side, providing the net active power required by OLEs: $\sum P_{i}$. In this way, each OLE may absorb/inject (load/generator) any active power into the AC scaled-down distribution system within the technical constraints imposed by the VSCs. The OLEs are connected to the following nodes: N3, N5, N6, N7, N8, N9, and N10 (subsytem 1), and N14 (subsystem 2). The active and reactive power references to the OLEs are set by a Signal Management System (SMS) which is detailed in the next subsection. 
A comprehensive description of this scaled-down system can be found in [25]. In addition, two new elements were incorporated with respect to the system described in [25] with the aim of integrating additional active control resources:

- Transformer with OLTC: The underlying idea of this feature is to represent the HV/MV transformers within the primary substations which are equipped with OLTCs to regulate the MV voltage. The transformer used for this purpose is a $400 \mathrm{~V} \pm 5 \% / 400 \mathrm{~V}, 100 \mathrm{kVA}$ equipped with a thyristor-based tap changer, as shown in Figure 4.

- DC link: This DC link, originally included in the benchmark distribution system [28], is incorporated between N8 and N14 as a suitable device to maximise the RES penetration, as stated previously. Although several topologies can be used to create a flexible loop between radially operated feeders [29], the DC link is based on conventional back-to-back VSCs rated at $400 \mathrm{~V}$ and $10 \mathrm{kVA}$. Note that the DC bus of the DC link is totally independent of the one shared by the OLEs and the balancing VSC.

The optimal setpoints for these two control assets are also managed by the SMS in a similar manner to that of the OLE power references.

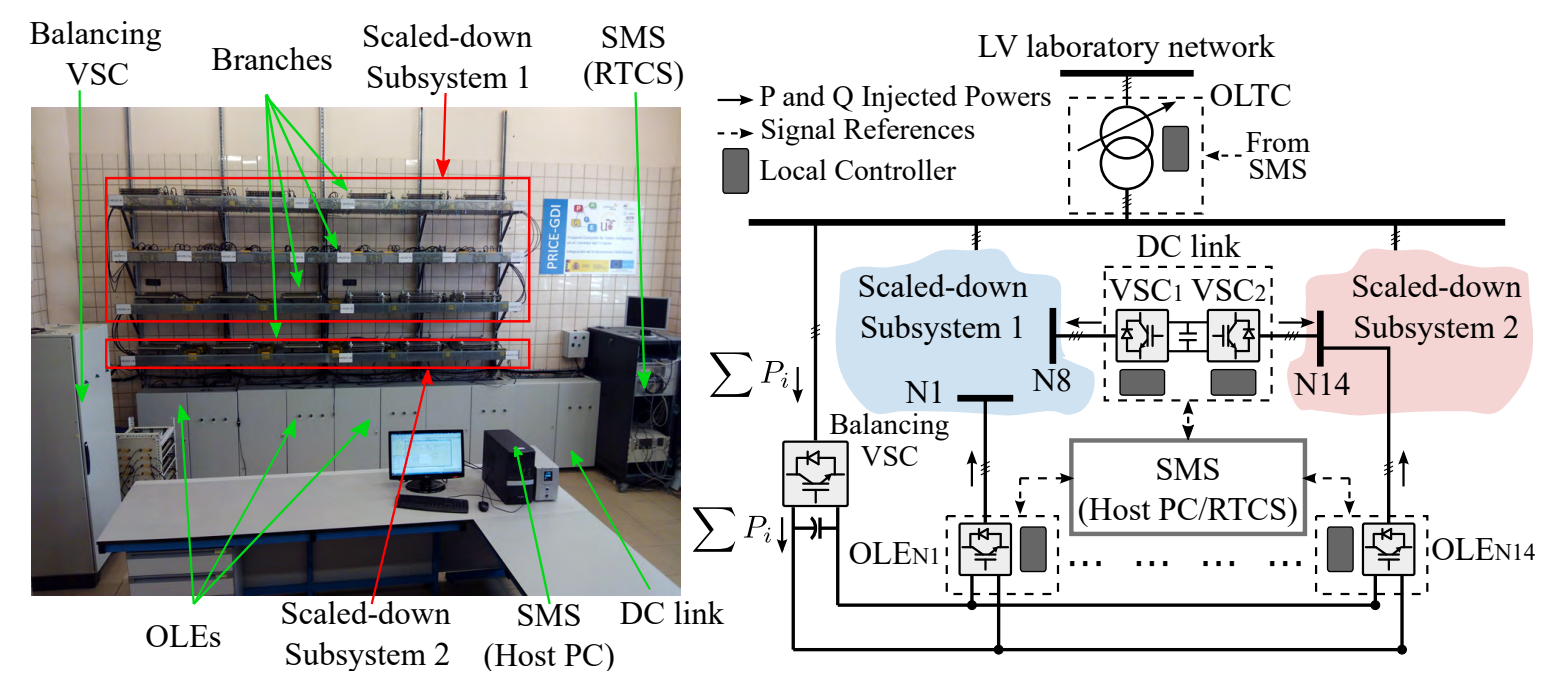

Figure 4. Left: Layout of the laboratory testbed. Right: One-line diagram of the updated testbed including the DC link and the transformer with the on-load tap changer (OLTC).

Table 1. Values of the resistors and reactors of each branch of the scaled-down network.

\begin{tabular}{cccc}
\hline Initial Node & End Node & Resistance $(\mathbf{m} \Omega)$ & Reactance $(\mathbf{m} \Omega)$ \\
\hline N1 & N2 & 60.00 & 39.25 \\
N2 & N3 & 25.00 & 15.75 \\
N3 & N4 & 5.00 & 3.25 \\
N4 & N5 & 10.00 & 3.25 \\
N5 & N6 & 25.00 & 7.75 \\
N6 & N7 & 5.00 & 1.50 \\
N7 & N8 & 25.00 & 7.75 \\
N8 & N9 & 5.00 & 1.50 \\
N9 & N10 & 10.00 & 3.25 \\
N10 & N11 & 5.00 & 1.50 \\
N11 & N12 & 10.00 & 3.25 \\
N3 & N8 & 10.00 & 6.25 \\
N12 & N13 & 60.00 & 62.50 \\
N13 & N14 & 25.00 & 15.75 \\
\hline
\end{tabular}




\subsection{Control Scheme and Communication System}

The control system is a two-level hierarchical structure, as shown in Figure 5. The first control level comprises the SMS, which is in charge of sending the references to the hardware components, whereas the second control level is composed of several LCs attached to the hardware devices (OLEs, DC link and OLTC) that are responsible for tracking these references.

The SMS performs two tasks in a sequential manner which can be summarised as follows:

- Offline tasks: They are carried out by a host PC and mainly consist of the configuration of the setpoint profiles. The OLE active and reactive daily power curves $\left(P_{i}^{\star}, Q_{i}^{\star}\right)$ are defined through two tools developed in the host PC [25]. Once these profiles have been determined, the daily setpoints of the DC link, $P_{\text {link }}^{o p t}$ and $Q_{v s c j}^{o p t}$, the reactive power injected by the RES, $Q_{w t, p v}^{o p t}$, and the optimal OLTC tap position, $t^{o p t}$, are automatically computed by the OPF described in Section 2. These setpoints and their computations are new features that are incorporated into the host PC with respect to [25]. Finally, all these data are compiled and uploaded to the Real-Time Control System (RTCS) for real-time operation.

- Online tasks: These are executed by the RTCS which is responsible for two undertakings. On the one hand, the RTCS is in charge of sending the setpoints to the second control level composed of the LCs attached to each hardware controllable component during the online operation according to the profiles previously determined in the offline tasks. On the other hand, the RTCS receives measurements from each each LC attached to the OLEs $\left(V_{i}, P_{i}\right.$ and $\left.Q_{i}\right)$, DC-link VSCs $\left(V_{v s c j}, P_{\text {link }}\right.$ and $\left.Q_{v s c j}\right)$ and the tap position of the transformer OLTC $\left(t^{o p t}\right)$. After processing this information, it provides real-time monitoring of the system which is displayed in the host PC.

The second level of the control system is composed of the LCs of each OLE, the DC-link VSCs, and the transformer OLTC which are implemented in Digital Signal Processors. These are in charge of tracking the setpoints sent by the RTCS during the online operation.

The communication infrastructure required to connect the centralised RTCS with the LCs is based on a $100 \mathrm{MBs}$ Ethernet local-area network as a physical layer that implements a communication protocol based on UDP/IP. Finally, an asynchronous communication protocol, TCP/IP, is implemented between the host PC and the RTCS.

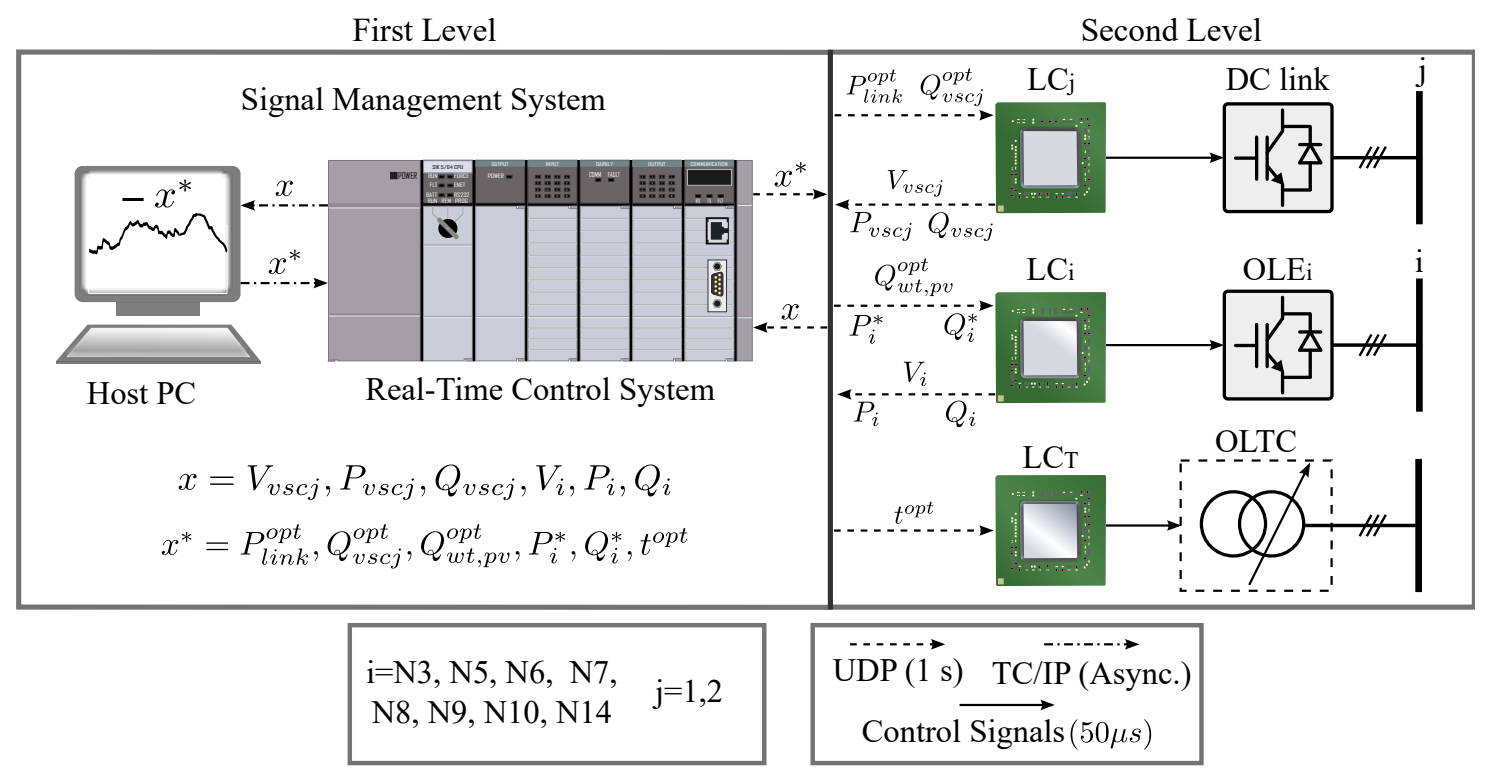

Figure 5. General control scheme of the testing environment. 


\section{Experimental Assessment of the Proposed Centralised Control}

This section describes the analysis of the performance of the centralised control on the scaled-down system under different test cases. These are evaluated through KPIs to quantify the influence of the considered control assets in high-RES active distribution networks.

\subsection{Definitions of Test Cases}

Table 2 shows the definitions of the designed test cases. The first case, $\mathrm{C} 1$, is the base case where no control assets are included in the distribution system and the OLTC is set in the central tap position. The subsequent test cases add the control assets in the centralised control in an incremental manner. In this way, it should be possible to quantify the impact that each control asset has on the system's performance.

Table 2. Definitions of test cases.

\begin{tabular}{l|cccc}
\hline Control Assets & C1 & C2 & C3 & C4 \\
\hline OLTC & & $\bullet$ & $\bullet$ & $\bullet$ \\
RES reactive power & & & $\bullet$ & $\bullet$ \\
DC link & & & & $\bullet$ \\
\hline
\end{tabular}

\subsection{Definitions of KPIs}

The following KPIs were selected to analyse the performance of the centralised control and its related control assets:

- Daily energy loss $\left(E_{\text {loss }} / \Delta E_{\text {loss }}\right)$ : This KPI measures the daily active energy loss in $\mathrm{kWh} /$ day, $E_{\text {loss, }}$ and the percentage of loss reduction with respect to the base case, $\mathrm{C} 1, \Delta E_{\text {loss }}$.

- Voltage violation $\left(T_{v v}\right)$ : This KPI evaluates the percentage of time during the day that which the nodal voltages are outside the technical limits [0.95-1.05 pu].

- Variation of nodal voltages $(\Delta V)$ : This index provides a global measurement of the daily voltage variations at the nodes of the network. It is computed as the average value of the difference between the maximum and minimum nodal voltages, measured in $\mathrm{pu}$,

$$
\Delta V=\frac{\sum_{\mathcal{N}}\left(V_{i}^{\max }-V_{i}^{\min }\right)}{N i}
$$

where $N i$ is the total number of network nodes.

- OLTC operation $\left(N_{O L T C}\right)$ : This KPI shows the number of OLTC operations that occur during the 24-h testing period.

- $\quad$ RES reactive power injection $\left(Q_{R E S}\right)$ : This index provides a global measurement of the RES collaboration to the network reactive power support. It is computed by dividing the average value of the reactive power injected by the RES during the 24-h period by the total number of RES,

$$
Q_{R E S}=\frac{\sum_{i, t} Q_{R E S_{i, t}}}{N_{t} \times N_{R E S}}
$$

where $Q_{R E S_{i, t}}$ is the reactive power injected by $R E S_{i}$ in period $t, N_{R E S}$ is the number of RES in the network, and $N_{t}$ is the number of time periods considered during the 24-h period.

- DC link load $\left(S L_{\text {link }}\right)$ : This evaluates the daily average load of the DC link during the day, and it is computed as

$$
S L_{\text {link }}=\frac{\sum_{j, t} S_{v s c j, t}}{N_{t} \times S_{D C l i n k}}
$$

where $S_{v s c j}$ is the apparent power of each VSC and $S_{D C l i n k}$ is the rated power of the DC link. 
- Transformer load $\left(T_{L}\right)$ : This represents the daily average load of the transformer as a percentage of its rated power, which can be computed as

$$
T_{L}=\frac{\sum_{t} S_{t}^{T}}{N_{t} \times S_{N}}
$$

where $S^{T}$ is the apparent power through the transformer and $S_{N}$ is the rated power of the transformer.

\subsection{Experimental Results}

The objective function proposed for the operation of high-RES active distribution networks is based on an operation with minimal technical losses. This section describes the evaluation of the previously described test cases, which involved the analysis of the following electrical magnitudes: power losses, nodal voltages, and current circulating at the primary substation transformer. In addition, the previously defined KPIs allowed the key magnitudes to be quantified in a comprehensive manner to assess the performance of the proposed control.

Table 3 shows the $E_{\text {loss }}$ for the studied test cases and the loss reduction with respect to the base case, $C 1, \Delta E_{\text {loss }}$, when the load and generation daily profiles presented in Section 3 were implemented into the testing platform. In the laboratory testbed, the 24-h profiles were scaled to the last $48 \mathrm{~min}$ and the duration of the tests was reduced.

Table 3. Key performance indices (KPIs) used for the evaluation of the test cases.

\begin{tabular}{l|cccc}
\hline & C1 & C2 & C3 & C4 \\
\hline$E_{\text {loss }} / \Delta E_{\text {loss }}(\mathrm{kWh} / \%)$ & $58.37 /-$ & $55.69 / 4.58$ & $50.17 / 16.33$ & $46.47 / 25.59$ \\
$Q_{R E S}(\mathrm{pu})$ & - & - & 0.117 & 0.095 \\
$T_{v v}(\%)$ & 38.69 & 0 & 0 & 0 \\
$N_{\text {OLTC }}$ & 0 & 2 & 4 & 2 \\
$\Delta V(\mathrm{pu})$ & 0.087 & 0.061 & 0.058 & 0.042 \\
$T_{L}(\%)$ & 24.95 & 24.43 & 20.62 & 20.20 \\
\hline
\end{tabular}

$\mathrm{C} 1$ presented the greatest daily power losses as no control assets were operating to act on the voltages and power flows to reduce the system losses. The introduction of the OLTC operation in C2 reduced energy losses by almost $5 \%$. The OLTC setpoint was computed in the OPF whose objective function was to reduce the total power losses in the network. Therefore, the tap was established in the $-5 \%$ position to increase the nodal voltages and to achieve the intended objective.

In test case C3, the RES reactive power capability was also included in the control. This caused the daily energy losses to be reduced by more than $15 \%$ with respect to $\mathrm{C} 1$. This occurred because the RES were able to provide reactive power to the system. Figure 6 shows the RES reactive power injected at nodes N3 and N8 with respect to their rated power levels for test cases C3 and C4. This is represented using violin plots which allow the distribution of any magnitude as well as its range of variation and frequency of occurrence to be visualized. Note that most of the time, which corresponds to the wider part of the violin plot, the RES were injecting reactive power corresponding to $20 \%$ of their rated power levels. This high RES reactive power injection was used to provide part of the reactive power demanded by the loads, thus avoiding the need to supply it from the primary substation, as shown in Figure 7. Note that the reactive power supplied from the primary substation in C3 was lower than 0.05 pu during the 24-h period, helping to reduce the energy losses.

The DC link integration in C4 further reduced the energy losses by up to $25 \%$ with respect to $\mathrm{C} 1$, as shown in Table 3. This device injected reactive power at the interconnected nodes N8 and N14 by means of VSC1 and VSC2 respectively during the 24-h period, as depicted in Figure 8. This power, added to the RES reactive power, led to almost zero reactive power being supplied from the primary substation, as shown in Figure 7. In this way, the energy losses reduced with respect to C3. An additional effect on the RES reactive power injections was observed. In C4, the RES did not to have 
to inject as much reactive power as in C3, as can be observed in Figure 6, even becoming zero in some nodes, like N8. This effect was quantified in a global manner with $Q_{R E S}$ collected in Table 3, where lower values for this KPI in C4 with respect to those in C 3 can be appreciated. Table 4 summarises the rated power and the reactive power injections of the RES units in C3 and C4. The second and third columns indicate the rated power of the RES used in the scaled-down system and the MV system respectively. The two last columns depict the maximum reactive power injected by the VSCs interfacing the RES units during the day in cases C3 and C4. These values refer to the rated power of each device. The RES connected to N5 injected the maximum amount of reactive power, reaching $31.45 \%$ of its rated power. With the current technology, these reactive power values are easily reachable due to the combined effect of two actions: (i) the VSC coupling reactance is becoming smaller by using LCL filters, and (ii) the VSC DC voltage is continuously increasing. This extends the VSC reactive power range.

Table 4. Rated power and maximum reactive power injection of the renewable energy source (RES) units in $\mathrm{C} 3$ and $\mathrm{C} 4$.

\begin{tabular}{ccccc}
\hline RES Connected to Bus & $S_{\text {rated }}(\mathbf{k V A})$ Scaled down System & $S_{\text {rated }}(\mathbf{M V A}) \mathbf{M V}$ System & $Q_{\text {max }}^{\text {C3 }}(\mathbf{p u})$ & $Q_{\text {max }}^{\text {C4 }}(\mathbf{p u})$ \\
\hline N3 & 12 & 12 & 0.3116 & 0.2584 \\
N5 & 12 & 12 & 0.3145 & 0.3144 \\
N6 & 12 & 12 & 0.2372 & 0.2372 \\
N7 & 7 & 7 & 0.0309 & 0.0309 \\
N8 & 12 & 12 & 0.2552 & 0.0100 \\
N9 & 12 & 12 & 0.1147 & 0.1147 \\
N10 & 16 & 16 & 0.1588 & 0.1588 \\
\hline
\end{tabular}
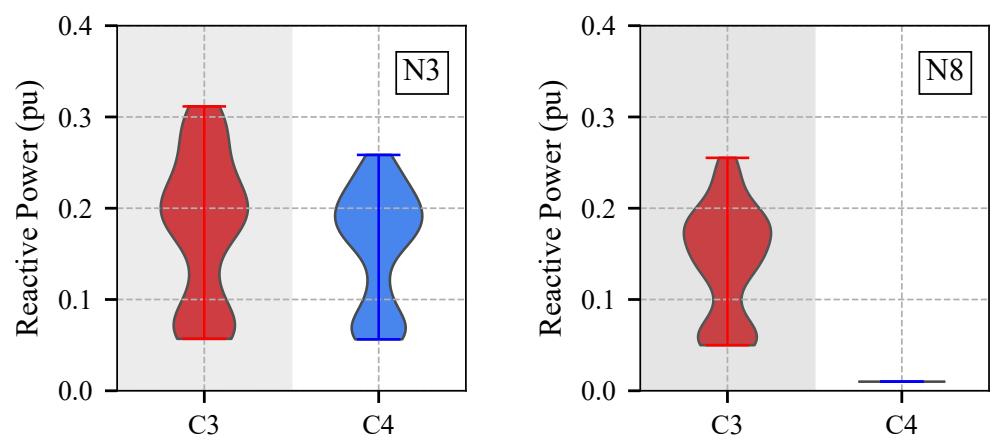

Figure 6. Violin plots of RES reactive power injections for test cases C3 and C4 at nodes N3 and N8.

Notice that the DC link also controlled the active power transferred from subsystem 1 to subsystem 2, as shown in Figure 8. Outside the period of high injection of RES active power $(0-10 \mathrm{~h}$ and 13-0 h), the DC link absorbed active power from N14 and injected it into N8. This meant that part of the load from subsystem 1 was powered by subsystem 2 which is less loaded and has shorter branches, helping to reduce the total power losses of the system. Conversely, within the hours of high RES active power injection, the active power flow was inverted in the DC link: VSC1 absorbed active power from subsystem 1 and it was injected by the VSC2 into subsystem 2 . In this way, part of the power generated by RES in subsystem 1 was transferred to feed the loads in subsystem 2 . Therefore, this active power was not supplied by the primary substation, thus reducing the current in this system and the energy losses.

Finally, note that the DC-link loading, $S L_{\text {link }}$, during the day was $49.4 \%$. This means that the DC link was used at half load and there is therefore still a wide margin to take advantage of its flexibility of operation. For example, the RES penetration in subsystem 1 could increase and still be managed by the current DC link. 


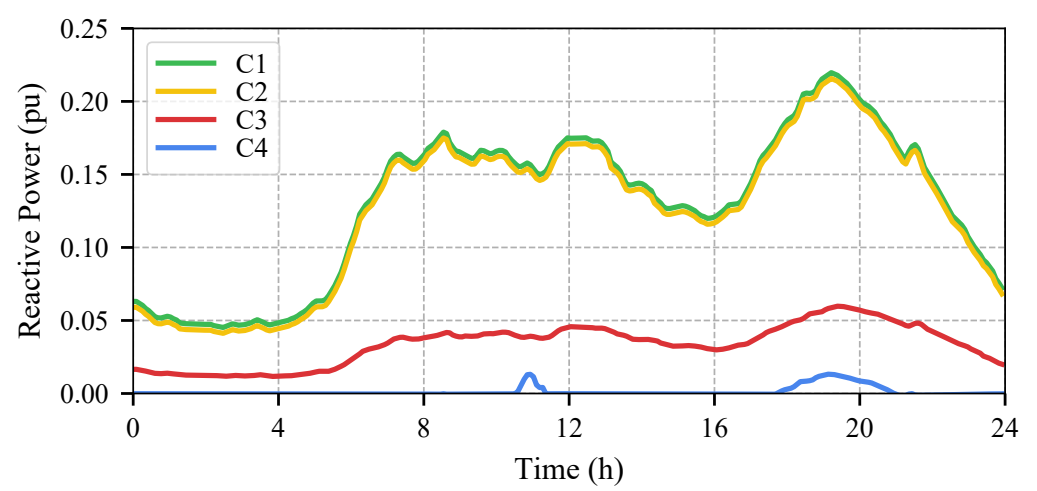

Figure 7. Reactive power flow through the primary substation for test cases C1-C4.
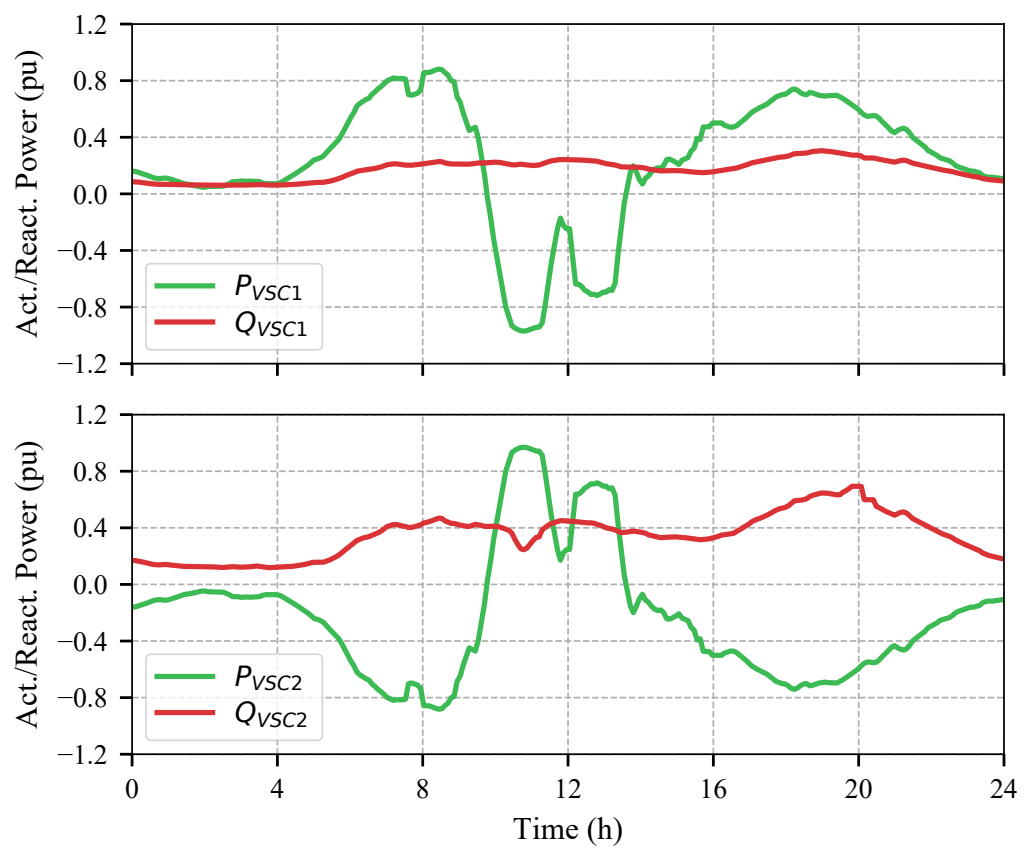

Figure 8. DC link active and reactive power daily profiles.

Figure 9 shows the 24-h nodal voltages at nodes N3, N6, N8, and N14 for the different test cases. These buses were selected to represent the behaviour of nodes nearby (N3) and far from (N6) the primary substation. In addition, nodes N8 and N14 were also included because they are the connection points of the DC link. The analysis of Figure 9 reveals that undervoltage situations-voltages below $0.95 \mathrm{pu}$ - exclusively occurred in the base case, C1, due to the lack of control assets operating in the network. This situation led to a very high $T_{v v}$ value in $\mathrm{C} 1$, as shown in Table 3 . These voltage violations were more severe at nodes N6 and N8 corresponding to subsystem 1 because of two reasons. First, subsystem 1 was more loaded than subsystem 2, as depicted in Figure 3, especially during the hours without RES generation. This caused greater current flows and, consequently, greater voltage drops along the lines. This effect was especially significant around 8 and $20 \mathrm{~h}$ when the RES generation was almost zero and the demand was peaking. 

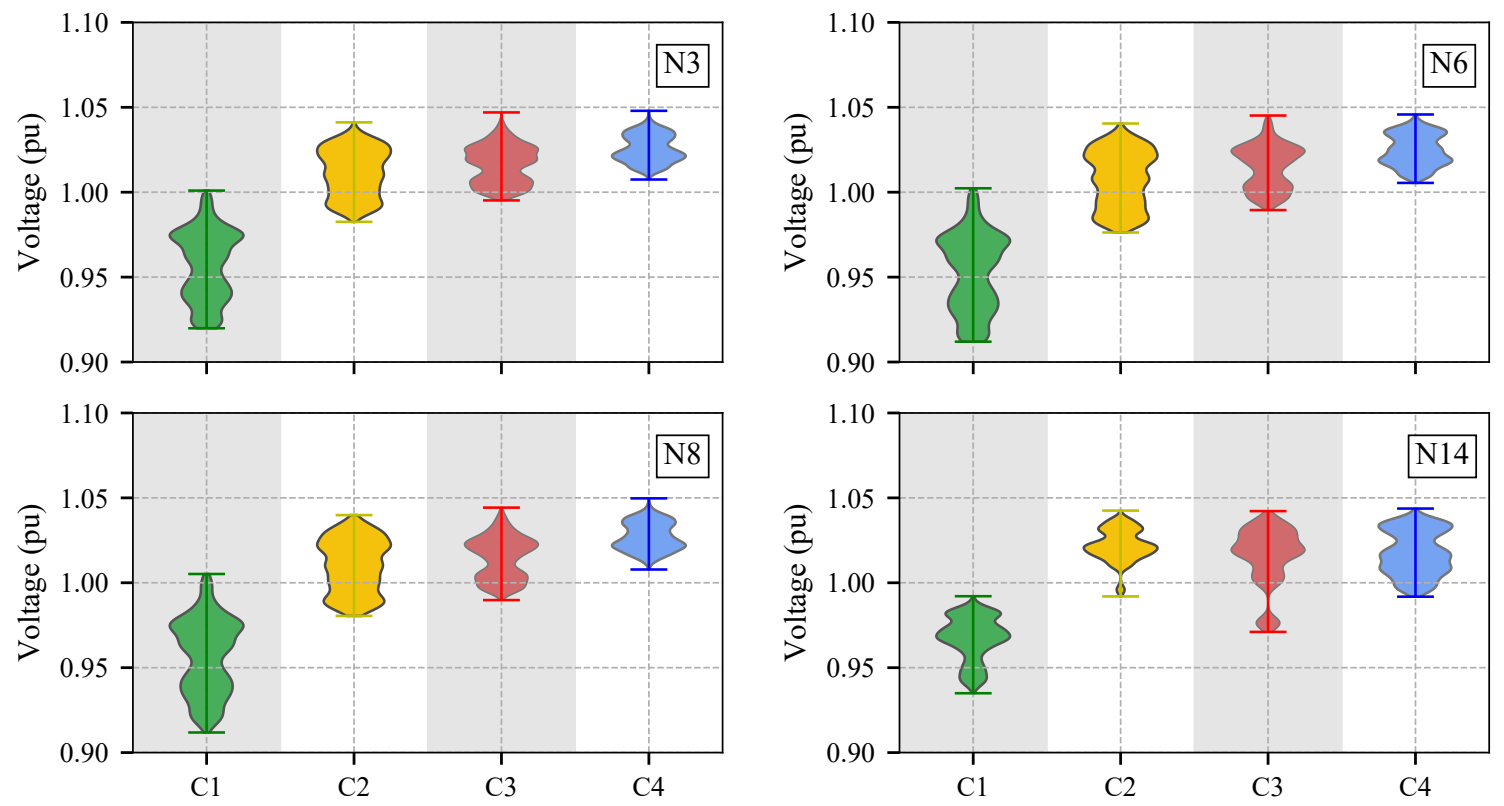

Figure 9. Violin plots of nodal voltages for test cases C1 to C4 at nodes N3, N6, N8, and N14.

The introduction of the OLTC in C2 pushed the voltages within the \pm 0.05 pu regulatory band around the rated voltage and, consequently, voltage violations were eliminated, as illustrated by its $T_{v v}$. In C2, the tap was established in the $-5 \%$ position for most of the day. However, according to the information provided in Table 3, two OLTC operations $N_{\text {OLTC }}$ (from $-5 \%$ to $0 \%$ position) over the 24 -h period were required to maintain the voltages within the limits. These changes occurred at around $11 \mathrm{~h}$ and $13 \mathrm{~h}$ when RES generation was maximum, as shown in Figure 3, and the network voltages were excessively high. The range of variation of nodal voltages $\Delta V$ was significantly reduced with respect to $\mathrm{C} 1$, as shown in Table 3. This effect can also be observed in Figure 9 where the violin plots are shortened, concentrating the nodal voltages within a narrower band. This trend was maintained in C3 due to the contribution of RES to the regulation of voltage with reactive power injections. In addition, it can be seen that the average voltage of nodes N3, N6, and N8 from subsystem 1 increased due to the local effect of the reactive power injections. As a consequence, additional OLTC changes $N_{\text {OLTC }}$ (from $-5 \%$ to $0 \%$ position) were required to maintain the voltages within the technical limits. This longer time of the tap within the $0 \%$ position caused lower voltages within subsystem 2 , as can be observed for the node N14 in Figure 9.

C4 incorporated the operation of the DC link between nodes N8 and N14 allowing the injection of additional reactive power into these nodes and active power transfer between both subsystems. This led to a minimum range of variation in the nodal voltages $\Delta V$ and maximum values of these in all the test cases. In fact, in C4, the voltages oscillated in a range between 1 and 1.05 pu over the 24-h period.

Figure 10 shows the daily evolution of the current circulating through the primary substation transformer for the studied test cases. This current reduced as the number of control assets increased. The analysis of $\mathrm{C} 4$ revealed that during some periods, the current was almost zero. This means that the generation of RES with adequate management by the control assets is enough to operate the system without the need of supplementary power from the primary substation. Finally, it is worth noting that the state of load of the transformer $T_{L}$ also progressively reduced in the subsequent test cases, as shown in Table 3. As a consequence, the benefits for the distribution system are clear in this respect: reduction of transformer losses, increment of useful life, and increase of the system loadability, which allows new investment in power assets to be deferred. 


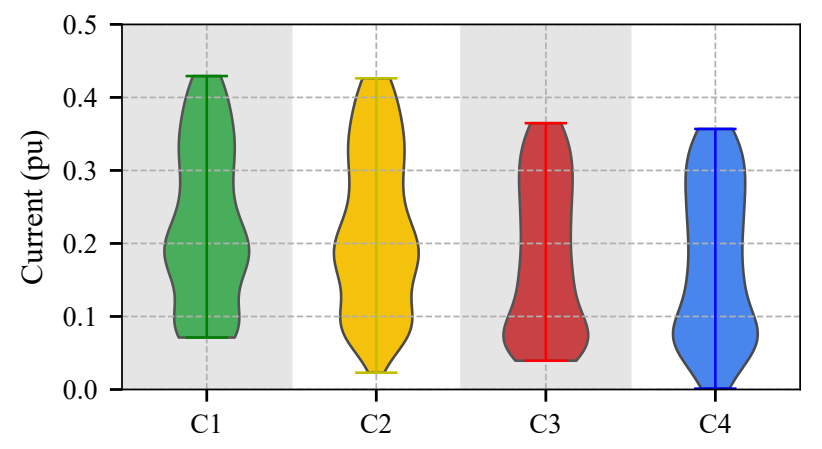

Figure 10. Violin plots of MV current at the primary substation transformer for test cases C1 to C4.

\section{Conclusions}

This paper assessed the benefits of a centralised controller for active distribution networks with high-RES penetration in an experimental manner. The paper proposed the optimization of the operation of the system through the minimization of active power losses through an OPF with the following control assets: (i) transformers equipped with OLTC, (ii) RES reactive power injections, and (iii) DC links. The assessment of the proposed centralised controlled was carried out on a laboratory scaled-down version of the MV network that was proposed by the CIGRE Task Force C06.04.02. This testing platform was described, including its main components and functionalities as well as the new control assets (transformer OLTC and DC link) which were incorporated into a previous version to improve its testing capabilities. The paper defined a comprehensive design of the testing procedure including some test cases involving different control assets and a set of KPIs to allow a quantintative comparison of performance. The obtained results revealed that a centralised control of high-RES active distribution networks may improve their operation. As a matter of fact, the obtained results, ranging from $15 \%$ to $25 \%$ of active power loss reduction, are consistent with those of similar works commented on in Section 1. Moreover, this improvement is significant for control assets which are commonly present in distribution networks, i.e., transformers with OLTC and RES reactive power injections. This enhancement could be even larger if uncommon but matured technologies, like DC links, were progressively introduced into the distribution business. This would increase the RES network hosting capacity, contributing to the decarbonization of our society.

Author Contributions: F.P.d.G.-L reviewed the state-of-the-art, designed the test cases and the control algorithms, was responsible for laboratory testing and discussion of the obtained results, and wrote part of the paper; M.B.-V. supported the laboratory testing, contributed to the analysis of the results and wrote part of the paper; A.M.-M. was responsible for the OPF definition, contributed to the analysis of results, and wrote part of the paper; J.M.M.-O. defined the KPIs, contributed to the discussion of results, and wrote part of the paper; J.L.M.-R. supported the OPF definition and practical implementation issues, and discussed the results of the paper.

Funding: The authors would like to acknowledge the financial support of the Spanish Ministry of Economy and Competitiveness under Grants ENE2015-69597-R, PCIN-2015-043 and ENE2017-84813-R.

Conflicts of Interest: The authors declare no conflict of interest.

\section{Abbreviations}

The following abbreviations are used in this manuscript:

ADMS Advanced Distribution Management System

CIGRE International Council on Large Electric Systems

DC Direct Current

DSO Distribution System Operator 


$\begin{array}{ll}\text { HV } & \text { High Voltage } \\ \text { IGBT } & \text { Insulated Gate Bipolar Transistor } \\ \text { KPI } & \text { Keys Performance Index } \\ \text { LC } & \text { Local Controller } \\ \text { LV } & \text { Low Voltage } \\ \text { MV } & \text { Medium Voltage } \\ \text { OLE } & \text { Omnimode Load Emulator } \\ \text { OPF } & \text { Optimal Power Flow } \\ \text { OLTC } & \text { On-Load Tap Changer } \\ \text { PV } & \text { Photovoltaic } \\ \text { RES } & \text { Renewable Energy Sources } \\ \text { RTCS: } & \text { Real-Time Control System } \\ \text { RTU } & \text { Remote Terminal Unit } \\ \text { SMS } & \text { Signal Management System } \\ \text { TSO } & \text { Transmission System Operator } \\ \text { VSC } & \text { Voltage Source Converter } \\ \text { WT } & \text { Wind Turbine }\end{array}$

\section{References}

1. Guerrero, J.M.; Blaabjerg, F.; Zhelev, T.; Hemmes, K.; Monmasson, E.; Jemei, S.; Comech, M.P.; Granadino, R.; Frau, J.I. Distributed Generation: Toward a New Energy Paradigm. IEEE Ind. Electron. Mag. 2010, 4, 52-64. [CrossRef]

2. Distributed Generation System Characteristics and Costs in the Buildings Sector; Technical Report; U.S. Energy Information Administration: Washington, DC, USA, 2013.

3. Pérez-Arriaga, I.; Knittel, C. Utility of the Future. An MIT Energy Initiative Response; Technical Report; Massachusetts Institute of Technology: Cambridge, MA, USA, 2016.

4. Power Distribution Planning Reference Book, 2nd ed.; CRC Press Book: Boca Raton, FL, USA, 2004.

5. Barker, P.P.; Mello, R.W.D. Determining the impact of distributed generation on power systems. I. Radial distribution systems. In Proceedings of the 2000 Power Engineering Society Summer Meeting (Cat. No.00CH37134), Seattle, WA, USA, 16-20 July 2000; Volume 3, pp. 1645-1656.

6. Walling, R.A.; Saint, R.; Dugan, R.C.; Burke, J.; Kojovic, L.A. Summary of Distributed Resources Impact on Power Delivery Systems. IEEE Trans. Power Deliv. 2008, 23, 1636-1644. [CrossRef]

7. Assessing the Impact of Low Carbon Technologies on Great Britain's Power Distribution Networks. 3 August 2012. Available online: https:/ / www.ofgem.gov.uk/publications-and-updates/assessing-impactlow-carbon-technologies-great-britains-power-distribution-networks (accessed on 5 April 2018).

8. Elkhatib, M.E.; El-Shatshat, R.; Salama, M.M.A. Novel Coordinated Voltage Control for Smart Distribution Networks With DG. IEEE Trans. Smart Grid 2011, 2, 598-605. [CrossRef]

9. Ueda, Y.; Kurokawa, K.; Tanabe, T.; Kitamura, K.; Sugihara, H. Analysis Results of Output Power Loss Due to the Grid Voltage Rise in Grid-Connected Photovoltaic Power Generation Systems. IEEE Trans. Ind. Electron. 2008, 55, 2744-2751. [CrossRef]

10. Molina-García, A.; Mastromauro, R.A.; García-Sánchez, T.; Pugliese, S.; Liserre, M.; Stasi, S. Reactive Power Flow Control for PV Inverters Voltage Support in LV Distribution Networks. IEEE Trans. Smart Grid 2017, 8, 447-456. [CrossRef]

11. Calderaro, V.; Galdi, V.; Lamberti, F.; Piccolo, A. A Smart Strategy for Voltage Control Ancillary Service in Distribution Networks. IEEE Trans. Power Syst. 2015, 30, 494-502. [CrossRef]

12. Karagiannopoulos, S.; Aristidou, P.; Hug, G. Hybrid approach for planning and operating active distribution grids. Transm. Distrib. IET Gener. 2017, 11, 685-695. [CrossRef]

13. Puerto Rico Electric Power Authority (PREPA). Minimum Technical Requirements (MTR) for Photovoltaic Generation (PV) Projects; Technical Report; Puerto Rico Electric Power Authority (PREPA): San Juan, Puerto Rico, 2012.

14. National Energy Regulator of South Africa (NERSA). Grid Connection Code for Renewable Power Plants (RPPS) Connected to the Electricity Transmission System or the Distribution System in South Africa; Technical Report; National Energy Regulator of South Africa (NERSA): Pretoria, South Africa, 2012. 
15. Alnaser, S.W.; Ochoa, L.F. Advanced Network Management Systems: A Risk-Based AC OPF Approach. IEEE Trans. Power Syst. 2015, 30, 409-418. [CrossRef]

16. Kryonidis, G.C.; Demoulias, C.S.; Papagiannis, G.K. A Nearly Decentralized Voltage Regulation Algorithm for Loss Minimization in Radial MV Networks With High DG Penetration. IEEE Trans. Sustain. Energy 2016, 7, 1430-1439. [CrossRef]

17. Kolenc, M.; Papič, I.; Blažič, B. Minimization of losses in smart grids using coordinated voltage control. Energies 2012, 5, 3768-3787. [CrossRef]

18. Alnaser, S.W.; Ochoa, L.F. Hybrid controller of energy storage and renewable DG for congestion management. In Proceedings of the 2012 IEEE Power and Energy Society General Meeting, San Diego, CA, USA, 22-26 July 2012; pp. 1-8.

19. Liu, X.; Aichhorn, A.; Liu, L.; Li, H. Coordinated Control of Distributed Energy Storage System with Tap Changer Transformers for Voltage Rise Mitigation Under High Photovoltaic Penetration. IEEE Trans. Smart Grid 2012, 3, 897-906. [CrossRef]

20. Barragan-Villarejo, M.; Marano, A.; García-López, F.P.; Mauricio, J.M.; Maza-Ortega, J.M. Coordinated control of distributed energy resources and flexible links in active distribution networks. In Proceedings of the International Conference on Renewable Power Generation (RPG 2015), Beijing, China, 17-18 October 2015; pp. 1-6.

21. Marano-Marcolini, A.; Villarejo, M.B.; Fragkioudaki, A.; Maza-Ortega, J.M.; Ramos, E.R.; de la Villa Jaén, A.; Delgado, C.C. DC Link Operation in Smart Distribution Systems With Communication Interruptions. IEEE Trans. Smart Grid 2016, 7, 2962-2970. [CrossRef]

22. Fazio, A.R.D.; Fusco, G.; Russo, M. Decentralized Control of Distributed Generation for Voltage Profile Optimization in Smart Feeders. IEEE Trans. Smart Grid 2013, 4, 1586-1596. [CrossRef]

23. Kulmala, A.; Alonso, M.; Repo, S.; Amaris, H.; Moreno, A.; Mehmedalic, J.; Al-Jassim, Z. Hierarchical and distributed control concept for distribution network congestion management. IET Gener. Transm. Distrib. 2017, 11, 665-675. [CrossRef]

24. Almasalma, H.; Claeys, S.; Mikhaylov, K.; Haapola, J.; Pouttu, A.; Deconinck, G. Experimental Validation of Peer-to-Peer Distributed Voltage Control System. Energies 2018, 11, 1304. [CrossRef]

25. Maza-Ortega, J.M.; Barragán-Villarejo, M.; García-López, F.d.P.; Jiménez, J.; Mauricio, J.M.; Alvarado-Barrios, L.; Gómez-Expósito, A. A Multi-Platform Lab for Teaching and Research in Active Distribution Networks. IEEE Trans. Power Syst. 2017, 32, 4861-4870. [CrossRef]

26. Romero-Ramos, E.; Gómez-Expósito, A.; Marano-Marcolini, A.; Maza-Ortega, J.M.; Martínez-Ramos, J.L. Assessing the loadability of active distribution networks in the presence of DC controllable links. IET Gener. Transm. Distrib. 2011, 5, 1105. [CrossRef]

27. Rudion, K.; Orths, A.; Styczynski, Z.A.; Strunz, K. Design of benchmark of medium voltage distribution network for investigation of DG integration. In Proceedings of the 2006 IEEE Power Engineering Society General Meeting, Montreal, QC, Canada, 18-22 June 2006; p. 6.

28. Benchmark Systems for Network Integration of Renewable and Distributed Energy Resources. Available online: http:/ / www.e-cigre.org/publication/575-benchmark-systems-for-network-integrationof-renewable-and-distributed-energy-resources (accessed on 2 November 2016).

29. Maza-Ortega, J.M.; Gomez-Exposito, A.; Barragan-Villarejo, M.; Romero-Ramos, E.; Marano-Marcolini, A. Voltage source converter-based topologies to further integrate renewable energy sources in distribution systems. IET Renew. Power Gener. 2012, 6, 435-445. [CrossRef]

(c) 2018 by the authors. Licensee MDPI, Basel, Switzerland. This article is an open access article distributed under the terms and conditions of the Creative Commons Attribution (CC BY) license (http://creativecommons.org/licenses/by/4.0/). 